\title{
SHORT PULSE X-RAY GENERATION BY LASER-THOMSON SCATTERING
}

\author{
F. Sakai, J. Yang, M. Yorozu, Y. Okada, T. Yanagida, Sumitomo Heavy Industries Ltd., 2-1-1 \\ Yatocho, Nishitokyo, Tokyo 188-8585, Japan \\ S. Ito, K. Takasago, A. Endo, The Femtosecond Technology Research Association, 5-5 Tokodai, \\ Tsukuba, Ibaraki 300-2635, Japan
}

\begin{abstract}
Short pulse X-rays were generated by Thomson scattering between a few picosecond electron bunches and 100fs Ti:Sapphire laser lights in a 90-degree and a headon interaction configurations. The electron beams $(0.5 \mathrm{nC} /$ pulse $)$ with $14 \mathrm{MeV}$ energy were focused to a beam radius of $100 \mu \mathrm{m}(\mathrm{rms})$. The energy and the pulse duration of the Ti:Sapphire laser used for the scattering experiments, were typically $100 \mathrm{~mJ}$ and $100 \mathrm{fs}$, respectively. The number of the X-ray photons generated at an interaction point was estimated to be $1.4 \times 10^{4}$ and $1.0 \times 10^{5}$ in the 90-degree and the head-on collisions, respectively. The pulse duration of the X-ray was calculated with the measured beam parameters of the electron and the laser beams to be $400 \mathrm{fs}$ and $3 \mathrm{ps}(\mathrm{rms}$.) in the 90-degree and the head-on collisions, respectively.
\end{abstract}

\section{INTRODUCTION}

A short pulse X-ray source is an important tool for studying the dynamics of the materials in the fundamental time scale. The development of femtosecond laser made it possible to study short time reactions in the femtosecond region. However, this optical probe is limited to studying extended electronic levels in solids, due to the low photon energy. An X-rays are important probes for studying the structure of solids through their interactions with core electronic levels in atoms. A femtosecond X-ray pulses can be used to observe structural dynamics on femtosecond time scale by utilizing various techniques, such as X-ray diffraction and extended X-ray absorption fine structure (EXAFS). The short pulse X-ray is also useful for industrial applications such as non-destructive inspection of high-speed rotating materials, and for medical imaging applications.

The generations of the X-ray through the Thomson scattering between relativistic electrons and short pulse laser lights have some advantages, such as good directional radiation, high brightness, wavelength tunability and a short pulse in the picosecond and femtosecond regions.

Femtosecond X-ray pulse was generated through the 90-degree Thomson scattering between tightly focused electron beam and femtosecond laser light[1]. Another group tried to generate the femtosecond X-ray pulse in the head-on collision configuration using femtosecond electron bunch compressed by a magnetic compressor [2].
$\mathrm{A} \mathrm{CO}_{2}$ laser was used as a scattering source to obtain high intensity X-ray [3].

Our group developed a stable high-brightness electron beam and a stable femtosecond high-power laser to apply to the short pulse X-ray for the industry and the medical imaging [4]. The results of the short X-ray pulse generation through the Thomson scattering are presented in this paper.

\section{THOMSON SCATTERING}

In Thomson scattering between a relativistic electron pulse and laser light, the energy of scattered photons under the Thomson limit $\left(\gamma \mathrm{Ep}<<\mathrm{mc}^{2}\right)$ is given by [5]

$$
E x=\frac{1-\beta \cos (\alpha)}{1-\beta \cos (\theta)} E p
$$

where Ex is the energy of the scattered X-ray photon, Ep is the energy of the incident laser light photon, $\alpha$ is the incident angle between the laser light and electron beam in the laboratory frame, $\theta$ is the emitting angle between the X-ray and the electron beam, $\beta$ is the ratio between $\mathrm{v}$ (the electron velocity and $\mathrm{c}$ (light velocity).

The number of the X-ray photons produced through Thomson scattering is given by

$$
\frac{d N x}{d t}=L \sigma
$$

where $\sigma$ represents the total cross section of Thomson scattering and, L is the luminosity determined by the collision geometry of the electron pulse and laser light pulse. The luminosity represents the density of the electrons and laser photons in the overlapping volume between the electron and laser light pulses.

The pulse length of an X-ray generated by Thomson scattering is determined by the interaction time between the electron and laser pulses. We assume that both the electron and laser light profiles satisfy the Gaussian profile. The pulse length of a scattering X-ray in the 90degree interaction is given by

$$
\alpha=\frac{\sigma_{L e} \sqrt{\sigma_{W e}^{2}+\sigma_{W p}^{2}+\sigma_{L p}^{2}}}{\sqrt{\sigma_{W e}^{2}+\sigma_{W p}^{2}+\sigma_{L e}^{2}+\sigma_{L p}^{2}}}
$$

where $\sigma_{\mathrm{Le}}$ and $\sigma_{\mathrm{We}}$ represent the length and beam width of electron pulse. $\sigma_{\mathrm{Lp}}$ and $\sigma_{\mathrm{Wp}}$ represent the length and beam width of laser light pulse. The pulse length of the $\mathrm{X}$-ray is almost equal to the one of the electron bunch in the headon collision and expressed by 
$\sigma x=\sigma_{L e}$

\section{EXPERIMENTAL SETUP}

The whole system is shown in Fig. 1. Electron beams are generated by a 1.6 cell S-band $(2856 \mathrm{MHz})$ photocathode RF gun driven by a picosecond UV laser. The electron beams are accelerated with a linear accelerator (linac) up to $14 \mathrm{MeV}$. The electron beams are focused with magnetic focusing lens at the interaction point. The electron beams are then bent with a bending magnet and damped at the lead block to prevent any Xray background caused by electron bombardment of the duct wall. A Ti:Sapphire tera-watt laser was used as the light source for the X-ray generation. The laser light interacted with the electron beam in configurations of the head-on and the 90 -degrees.

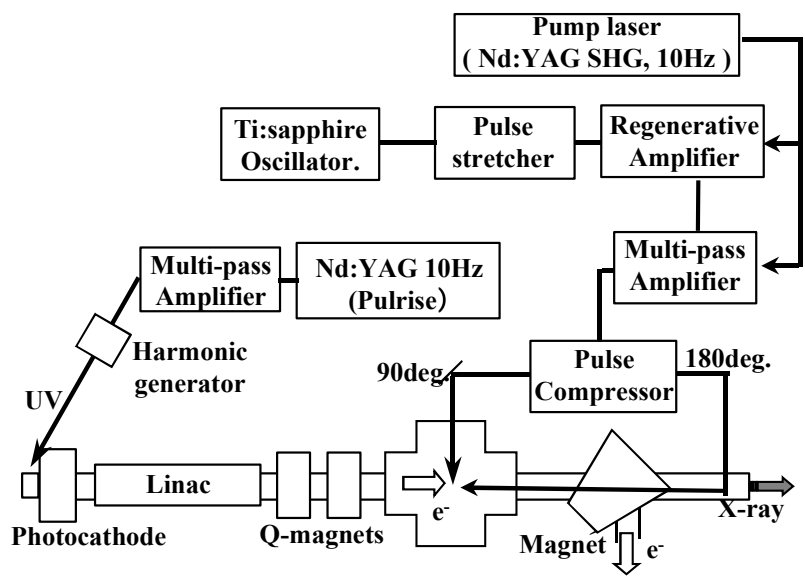

Figure 1 Experiment System

The 1.6 cell photocathode RF gun for high duty operation was developed by the BNL/KEK/Sumitomo collaboration [6]. The quantum efficiency of a cathode made of $\mathrm{Cu}$ was $1.5 \times 10^{-4}$. More than $2 \mathrm{nC} /$ pulse was obtained. The energy dispersion $(\delta \mathrm{E} / \mathrm{E})$ was $0.3 \%$ (rms.) at $14 \mathrm{MeV}$. The normalized rms. emittance of the electron beams with a $0.5 \mathrm{nC} /$ pulse was $2 \pi \mathrm{mm}$.mrad

The drive laser for the RF photocathode gun is important for electron beam stability. An LD pumped Nd:YAG laser was developed [7]. This laser consists of a passive mode-locked oscillator with a semiconductor saturable absorber mirror (SESAM), a regenerative amplifier and a multi-pass post amplifier, and a frequency conversion part. The frequency of the oscillator is 119 $\mathrm{MHz}$, equal to $1 / 24$ of the $2856 \mathrm{MHz}$ S-band RF. A timing stabilizer minimized the timing jitter of the oscillator by adjusting the laser cavity length. The timing jitter between the reference RF signal and the output of the oscillator was controlled to within $0.5 \mathrm{ps}$. The UV $(266 \mathrm{~nm})$ pulse energy was more than $200 \mu \mathrm{J}$. The fluctuation of the UV laser pulse energy was measured to be $3-5 \%$ (rms.).

The 1 TW femtosecond Ti:Sapphire laser system was developed [8] as a scattering light source. The mode- locked Ti:Sapphire laser oscillator generated $50 \mathrm{fs}$ pulses at the repetition rate of $119 \mathrm{MHz}$. The frequency of the laser oscillation was controlled by the same method as the drive laser for the gun. $2.5 \mathrm{~nJ}$ laser pulses from the oscillator were stretched in a pulse stretcher up to 400ps. The stretched pulses were amplified in a regenerative amplifier and then in a multi-pass post amplifier. The output pulse energy in the regenerative amplifier and the multi-pass amplifier were $10 \mathrm{~mJ}$ and $270 \mathrm{~mJ}$. The amplified pulses were delivered to a pulse compressor located near the interaction chamber. The pulse compressor consisted of a pair of gratings, with one grating installed in vacuum. The compressed pulse energy and the pulse duration were typically $100 \mathrm{~mJ}$ and $100 \mathrm{fs}$ (FWHM).

A micro-channel plate (MCP) used as an X-ray detector was located $2 \mathrm{~m}$ from the interaction point. The MCP, which has very low sensitivity for higher energy X-ray and a fast decay time constant in ns region, was chosen to prevent the high energy X-ray background produced by the field emission electrons at the linac and the gun. The diameter of the detector was $18 \mathrm{~mm}$, which corresponded to $4.5 \mathrm{mrad}$ collection angle from the detector. The calibrated gain of the MCP was $2.6 \mathrm{pC} /$ photoelectron. The quantum efficiency of the detector was also calibrated with radioisotope sources. The detector efficiency was found to be $5 \%$ and $8 \%$ for X-rays with $4.6 \mathrm{keV}$ and $2.3 \mathrm{keV}$ energy, respectively [9],[10].

\section{RESULTS AND DISCUSSION}

The electron and the laser beam parameters in the headon and 90-degree configurations are shown in Table I. The electron beam size at the interaction point was measured with a $100 \mu \mathrm{m}$ thick phosphor screen and a CCD camera. The laser beam size was also measured at the screen. The charge of the electron bunch was measured by a current transformer with a fast response time. The laser pulse duration was measured by the standard correlation method. The pulse duration of the electron bunch was measured by the Thomson scattering in the 90-degree configuration by changing the time delay between the electron and the laser beams.

Figure 2 shows the MCP signals, the scattering X-ray signal and the background signal, in the 90-degree configuration. Figure 3 shows a relation between the laser energy and the scattering X-ray photons. The energy of the scattering X-ray photons calculated by eq. (1) was 2.3 and $4.6 \mathrm{keV}$ in the 90 -degree and head-on collisions. The number of X-ray photons deposited on the MCP was estimated from the gain and the quantum efficiency of the detector. The number of X-ray photons at the interaction point in the 90-degree collision was estimated from the collection angle of the detector to be $1.4 \times 10^{4}$ photons/pulse. The numbers of total photons calculated from eq. (2) using the measured beam parameters was $1.2 \times 10^{5}$ photons/pulse. The pulse duration of X-ray was estimated using eq. (3) to be $400 \mathrm{fs}$ ( $\mathrm{rms}$.). The number of $\mathrm{X}$-rays in the head-on collision at the interaction point 
was $1.0 \times 10^{5}$ (measured) and $1.0 \times 10^{6}$ photons/pulse (calculated). The number of observed X-rays was one order lower than that of the theoretical calculations. The reasons for this are now under investigation.

\section{CONCLUSION}

The generation of femtosecond X-ray pulses was demonstrated through Thomson scattering in a 90-degree collision between relativistic high brightness electron beams and tera-watt femtosecond laser beams. The number of X-ray photons generated at an interaction points was estimated to be $1.4 \times 10^{4}$ and $1.0 \times 10^{5}$ at the 90 degree and the head-on collisions. The pulse width of the X-ray beam was calculated with the measured beam parameters of the electron and the laser beams to be 400 fs and 3ps (rms.) at the 90-degree and the head-on collisions, respectively.

We also plan to increase the X-ray energy from a few $\mathrm{keV}$ to $30 \mathrm{keV}$ to utilize this $\mathrm{X}$-ray source in practical applications, e.g. non-destructive inspection of defects in high-speed rotational metals and the medical imaging. We will increase the electron energy up to more than $35 \mathrm{MeV}$ and the output power of the laser for the scattering up to $1 \mathrm{~J} /$ pulse in a couple of years.

\section{ACKNOWLEDGEMENT}

This work was performed under the management of a technological association, the Femtosecond Technology Research Association (FESTA), supported by the New Energy and Industrial Technology Development Organization (NEDO).

\section{REFERENCES}

[1] R.W.Schoenlein, et al., Science 274, (1996) 236

[2] S. Kashiwagi et al., Nucl. Instr. \& Meth. A455 (2000) 36

[3] H.Kotaki et al., Nucl. Instr. \& Meth. A455 (2000) 166

[4] A. Endo et al., SPIE 4144 (2000) 16

[5] J.Yang, M. Washio, A. Endo and T. Hori, Nucl. Instr. \& Meth. A428, (1999) 556

[6] F. Sakai et al., Proc. of the 11th Sympo on Accel. Sci. and Tech., Harima,473(1997)

[7] F. Sakai et al., Proc. of 1999 PAC, New York, 2036(1999)

[8] S. Ito et al., in Tech. Dig. Advanced Solid-State Lasers, Seattle, (2001), Paper MF5

[9] E. Kellop, P. Henry, S. Murray and L. V. Speybroeck, Rev. Sci. Instr. 47, (1976) 282

[10] G. W. Fraser, Nucl. Instr. \& Meth. 195, (1982) 523
Table 1: Parameters of the electron and the laser beams

\begin{tabular}{|l|c|c|}
\hline & 90 deg. & 180 deg. \\
\hline Electron & & \\
\hline Energy(MeV) & 14 & 14 \\
\hline Charge(nC/pulse) & 0.5 & 1.2 \\
\hline Beam size( $\mu$ m:rms) & 100 & 120 \\
\hline Pulse duration(ps:rms) & 3 & - \\
\hline Laser & & \\
\hline Wavelength(nm) & 800 & 800 \\
\hline Pulse energy $(\mathrm{mJ} / \mathrm{pulse})$ & 85 & 85 \\
\hline Pulse duration $(\mathrm{fs}: \mathrm{rms})$ & 100 & 54 \\
\hline Beam size $(\mu \mathrm{m}: \mathrm{rms})$ & 20 & 180 \\
\hline
\end{tabular}

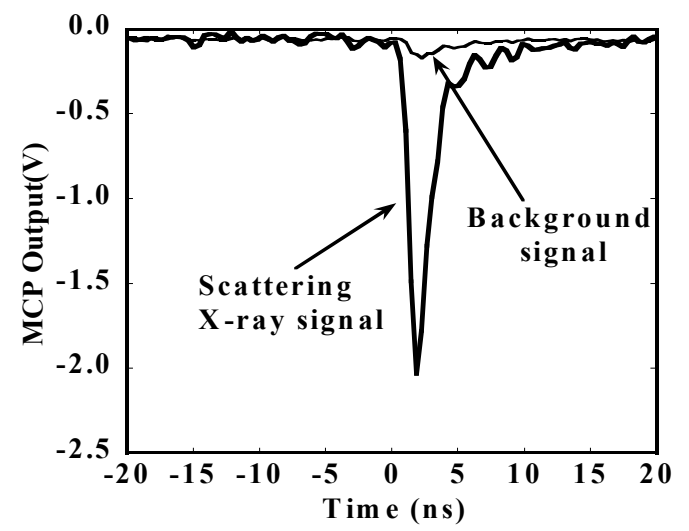

Figure 2 MCP signals of X-ray and Background Two solid lines show the MCP signals when the femtosecond laser was on and off.

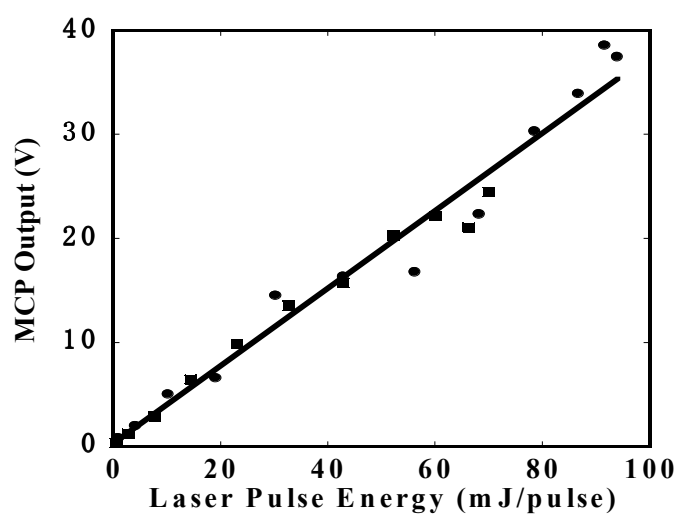

Figure 3 X-ray intensity vs. Laser energy Solid line: a least squire fitting with a linear function 